\title{
Évaluation de la résistance de dix porte-greffes d'agrumes résistants à la tristeza vis-à-vis du déficit hydrique
}

\author{
Lhou BeNIKEN ${ }^{1 *}$, Mohamed BeQQALI ${ }^{1}$, Rachid DAHAN ${ }^{1}$, Rachid BeNKIRANE ${ }^{2}$, Fatima Ezahra OMARI ${ }^{1}$, Abdelkadir BenAzouz ${ }^{1}$, \\ Hamid Benyahia ${ }^{1}$, Fatima Gaboun ${ }^{1}$
}

\author{
${ }^{1}$ Inst. Natl. Rech. Agron. INRA \\ Maroc, CRRA Kenitra, \\ BP 7567, Kenitra, Maroc \\ beniken_lhou@yahoo.fr \\ 2 Lab. Bot. Prot. Plantes, \\ Univ. Ibn Tofail, \\ Fac. Sci. Kenitra, Maroc
}

\section{Evaluation of resistance of ten citrus rootstocks resistant to tristeza regarding the water deficit.}

Abstract - Introduction. In Morocco, citriculture faces up an increasing combination of biotic (Citrus tristeza virus (CTV) / Phytophthora spp.) and abiotic stresses (salinity, alkalinity and drought). Most of these constraints should be individually managed by adapted rootstock choice. However, the spread of CTV in the Mediterranean Basin will soon prohibit the use of the traditional sour orange rootstock, which currently provides sufficient tolerance to salinity and alkalinity. Therefore, the selection of new citrus rootstocks becomes urgent. Our study sought to investigate the effect of different water regimes on growth of ten citrus rootstocks: citrange Carrizo (France), citrange Carrizo (Morocco), citrange Troyer, citrange Yuma, Citrus volkameriana; two Poncirus trifoliata of different origins, citrumelo, Mand. Sunki $\times$ Poncirus trifoliata, and Poorman. Materials and methods. For each rootstock, three months old seedlings were grown in plastic pots $(0.5 \mathrm{~L})$ in a greenhouse at El Menzeh, INRA, Morocco; they were subjected to three water regimes [(100\%, 75\% and 50\%) moisture field capacity ( $\mathrm{Hcc}$ ) of the substrate]. The experiment was arranged in a split-plot design with three treatments. The morphological parameters measured were the plant height and dry and fresh matter accumulation. Physiological parameters studied were the Relative Water Content, transpiration rates and chlorophyll, soluble sugars and proline contents. Results. The morphological and physiological parameters were affected by the intensity of water stress applied. However, rootstocks behaved differently regarding to this constraint. Water stress induced an increase in the proline and soluble sugars content in the rootstock leaves. Conclusion. Water stress significantly affects the physiological and morphological characteristics of citrus rootstocks and, in conditions of extreme water deficit (50\% Hcc), a differential behavior of the rootstock was found. Based on our results, the rootstocks Carrizo citrange (Morocco) and Poorman present the best overall ability to withstand water stress.

Morocco / Citrus / rootstocks / drought stress / drought resistance / proline / carbohydrate content

\section{Évaluation de la résistance de dix porte-greffes d'agrumes résistants à la tristeza vis-à-vis du déficit hydrique.}

Résumé - Introduction. Au Maroc, les agrumes sont confrontés à une combinaison de stress biotiques [virus de la tristeza (CTV) / Phytophthora] et abiotiques (salinité, alcalinité et sécheresse). La plupart de ces contraintes doivent être gérées individuellement par le choix d'un porte-greffe adapté. Cependant, la propagation de la CTV dans le bassin méditerranéen va bientôt interdire l'utilisation du bigaradier, porte-greffe traditionnel, qui offre actuellement une tolérance suffisante à la salinité et l'alcalinité. Par conséquent, la sélection de nouveaux porte-greffes d'agrumes devient pressante. Notre étude a cherché à étudier l'effet de différents régimes hydriques sur la croissance de dix portegreffes d'agrumes : citrange Carrizo (France), citrange Carrizo (Maroc), citrange Troyer, citrange Yuma, Citrus volkameriana; deux Poncirus trifoliata d'origine différente, citrumelo, Mand. Sunki $\times$ Poncirus trifoliata, et Poorman. Matériel et méthodes. Pour chaque porte-greffe, des plants âgés de trois mois ont été cultivés en pots de plastique $(0,5 \mathrm{~L})$ dans une serre à El Menzeh, INRA, Maroc ; ils ont
* Correspondance et tirés à part

Reçu le 11 juin 2010

Accepté le 28 septembre 2010

Fruits, 2011, vol. 66, p. 373-384 (C) 2011 Cirad/EDP Sciences All rights reserved DOI: $10.1051 /$ fruits/2011053 www.fruits-journal.org

RESUMEN ESPAÑOL, p. 384 du substrat]. L'expérimentation a été disposée en un split-plot à trois traitements. Les paramètres morphologiques mesurés ont été la hauteur de la plante et l'accumulation de matière sèche et fraîche. Les paramètres physiologiques étudiés ont été la teneur relative en eau, le taux de transpiration et les teneurs en chlorophylle, sucres solubles et proline. Résultats. Les paramètres morphologiques et physiologiques ont été affectés par l'intensité du stress hydrique appliqué. Cependant, les portegreffes se sont comportés différemment vis-à-vis de cette contrainte. Le stress hydrique a induit une augmentation de la teneur en proline et en sucres solubles au niveau des feuilles des porte-greffes. Conclusion. Le stress hydrique affecte d'une manière significative les caractéristiques physiologiques et morphologiques des porte-greffes d'agrumes et, en conditions de déficit hydrique extrême ( $50 \%$ Hcc), un comportement différentiel des porte-greffes a été constaté. Sur la base des résultats que nous avons obtenus, ce sont les porte-greffes citrange Carrizo (Maroc) et Poorman qui présenteraient globalement les meilleures aptitudes à résister aux conditions de stress hydrique.

Maroc / Citrus / porte-greffe / stress dû à la sécheresse / résistance à la sécheresse / proline / teneur en glucides 


\section{Introduction}

Au Maroc, la plupart des vergers agrumicoles sont greffés sur le bigaradier (Citrus aurantium L.) en raison de ses capacités d'adaptation aux différents types de sol, sa meilleure compatibilité avec la plupart des variétés utilisées et de sa bonne résistance aux maladies, en particulier à Phytophtora $[1,2]$. En revanche, l'association des variétés avec ce porte-greffe sont reconnues sensibles à la tristeza, maladie virale dévastatrice des agrumes ; ce qui constitue une menace permanente pour le verger agrumicole méditerranéen. De ce fait, la recherche de porte-greffes de remplacement résistants à la tristeza s'imposerait.

Au Maroc, parmi les caractéristiques recherchées pour de nouveaux portegreffes de relais au bigaradier figure la résistance et/ou la tolérance à la sécheresse. En effet, la plupart des zones du bassin méditerranéen où l'on cultive les agrumes sont sujets à des changements des facteurs abiotiques et plus particulièrement au manque d'eau, ce qui rend nécessaire le recours à l'irrigation. Or, il se trouve que les terrains irrigués présentent des problèmes de salinité importants car l'eau utilisée pour l'irrigation contient des sels qui augmentent l'évaporation et favorisent la désertification. Il s'ensuit des conditions de stress chroniques qui peuvent affecter aussi bien la croissance que la production des agrumes. Dans ce contexte, plusieurs études ont montré que le stress hydrique affecte négativement la croissance des plantes via plusieurs processus : la réduction de l'activité photosynthétique, le changement du statut hydrique, la fermeture des stomates, la réduction de la surface foliaire et l'augmentation de la température foliaire [3, 4].

Les agrumes sont considérés comme des cultures exigeantes en eau (entre [(900 et 1200) $\mathrm{mm} \cdot \mathrm{an}^{-1}$ ) [5]. La sélection de portegreffes d'agrumes résistant au stress hydrique permettrait donc la sauvegarde des agrumes du Bassin méditerranéen et le développement d'une agriculture durable en raison d'une demande d'irrigation plus faible.
Plusieurs études ont montré une grande variabilité de tolérance des porte-greffes et d'associations [variétés / porte-greffes] visà-vis de la contrainte hydrique. Selon Yoshimi et al., le porte-greffe aurait une grande influence sur le flux de la sève brute chez la mandarine Shirakawa Satsuma [6]. Ces auteurs ont rapporté également que le porte-greffe pouvait contribuer significativement à une économie d'eau d'irrigation et tolérer des conditions de sécheresse en culture d'agrumes.

Les différentes espèces et variétés d'agrumes utilisées comme porte-greffes ont des comportements différents vis-à-vis du manque d'eau ou du degré de salinité du terrain. Leurs comportements oscillent entre la forte sensibilité et la tolérance. Malheureusement, les génotypes qui semblent mieux tolérer les stress abiotiques sont souvent les plus sensibles aux maladies. Par exemple, le mandarinier Cleopatra (Citrus reshni) est considéré comme le mieux résistant à la salinité, tandis que Poncirus trifoliata est reconnu résistant à la tristeza (maladie virale), à Phytophtora et aux nématodes, mais il est très sensible au sel et aux terrains calcaires ; il a un système racinaire très superficiel et est donc exposé au risque de stress hydrique.

Il est admis que le comportement des plantes vis-à-vis du stress hydrique diffère d'une façon significative à différents niveaux de l'organisation : morphologique, physiologique et biochimique ; il est fonction de l'intensité et de la durée du stress ainsi que des espèces végétales concernées et du stade de croissance du plant [7-9]. L'étude de la réaction des plantes vis-à-vis de ce stress est d'une importance primordiale car elle permet de sélectionner des génotypes résistants à cette contrainte [9$11]$.

Chez les agrumes, le choix d'un portegreffe tolérant à ce stress abiotique serait un moyen efficace pour pallier le problème du manque d'eau et de sa salinité. Notre expérimentation a permis d'évaluer, vis-à-vis du stress hydrique, dix porte-greffes résistants à la tristeza, dont neuf récemment introduits au Maroc. 


\section{Matériel et méthodes}

\subsection{Matériel végétal et conduite des traitements}

Neuf porte-greffes d'agrumes introduits au Maroc à partir du germplasm agrumes de la SRA de San Giuliano (Corse, France) en remplacement du bigaradier et un citrange Carrizo déjà présent localement ont été utilisés (tableau I). Les semences ont été récoltées à partir de la collection du germplasm et du parc semencier des agrumes du domaine expérimental El Menzeh de l'Institut national de la recherche agronomique (INRA) de Kenitra au Maroc.

Les semences ont été stérilisées par immersion dans une solution d'hypochlorite de sodium à $15 \%$ pendant 15 min et rincées à l'eau distillée. Les semis ont été effectués dans des germoirs contenant $100 \%$ de tourbe. Après germination, les plantules homogènes ayant trois à quatre feuilles ont été repiquées dans des pots en plastique de $500 \mathrm{~mL}$ contenant un substrat composé d'un mélange de $1 / 3$ de tourbe et $2 / 3$ de sable, présentant un $\mathrm{pH}$ de 7,09 et contenant $2,25 \%$ de matière organique, $267,57 \mathrm{mg}$ $\mathrm{P}_{2} \mathrm{O}_{5} \cdot \mathrm{kg}^{-1}$ et $268,11 \mathrm{mg} \mathrm{K} 2 \mathrm{O} \cdot \mathrm{kg}^{-1}$. Après une période d'adaptation de 1 mois, les plants des différents porte-greffes ont été disposés en un split-plot avec le facteur dose d'irrigation comme traitement et le facteur porte-greffe comme sous-traitement. Les plants ont été irrigués trois fois par semaine et durant 60 jours. L'essai a été installé sous serre au domaine expérimental El Menzeh (INRA, Kenitra) pendant 3 mois (juin à aout 2008) ; les températures maximales moyennes pendant ces mois ont été respectivement de $(25,93,29,04 \text { et } 27,8)^{\circ} \mathrm{C}$ et les températures minimales respectivement de $(13,61,16,01$ et 16,2$)^{\circ} \mathrm{C}$. Les doses d'arrosages appliquées ont représenté une proportion de l'humidité à la capacité au champ (Hcc) du substrat. Les trois niveaux de stress ainsi testés ont été de $100 \%$ Hcc ( $\mathrm{T}_{0}$, traitement témoin), $75 \%$ $\operatorname{Hcc}\left(\mathrm{T}_{1}\right)$ et $50 \% \operatorname{Hcc}\left(\mathrm{T}_{2}\right)$.

\subsection{Mesures effectuées}

\subsubsection{Paramètres morphologiques}

À la fin de l'expérience, pour chaque traitement d'irrigation appliqué et chaque porte-

$\begin{aligned} & \text { Tableau I. } \\
& \text { Porte-greffes d'agrumes testés au } \\
& \text { résistance à des régimes hydriques différents. }\end{aligned}$
\begin{tabular}{lc} 
Identification du germplasm agrumes & Nom du porte-greffe \\
SNRA, France & \\
\hline ICVN 0110181 & Citrange Carrizo (France) \\
- & Citrange Carrizo (Maroc) \\
ICVN 0110197 & Citrange Troyer \\
ICVN 0110202 & Citrange Yuma \\
ICVN 0110141 & Citrumelo \\
ICVN 0110025 & Citrus volkameriana \\
ICVN 0110210 & Mand. Sunki $\times$ Poncirus trifoliata \\
ICVN 0110107 & Poncirus trifoliata (I) \\
ICVN 0110444 & Poncirus trifoliata (II) \\
ICVN 0110218 & Poorman
\end{tabular}

greffe testé, les racines et les feuilles ont été séparées de la tige. La longueur finale de la tige a été mesurée au moment de l'arrachage et la biomasse fraîche totale ainsi que celle des parties aériennes et radiculaires ont été alors déterminées par pesée immédiate. La biomasse sèche totale et celle des différents organes (tige, feuilles et racines) ont été déterminées après passage à l'étuve à $80^{\circ} \mathrm{C}$ durant $48 \mathrm{~h}$.

\subsubsection{Paramètres physiologiques}

\subsubsection{La teneur relative en eau}

Une feuille par traitement et par porte-greffe a été coupée avec son pétiole et pesée immédiatement pour déterminer le poids frais $\left(\mathrm{P}_{\mathrm{f}}\right)$. Les feuilles pesées ont été mises dans de l'eau distillée (pétiole submergé) à l'obscurité pendant $24 \mathrm{~h}$ puis de nouveau pesées afin d'obtenir leur poids de saturation $\left(\mathrm{P}_{\text {sat }}\right)$. Ensuite, les échantillons ont été mis en étuve pendant 24 h à $80^{\circ} \mathrm{C}$; le poids $\sec \left(\mathrm{P}_{\mathrm{sec}}\right)$ a alors été mesuré et la teneur relative en eau (RWC) a été calculée selon la formule $\left\{\mathrm{RWC}(\%)=100 \times\left[\left(\mathrm{P}_{\mathrm{f}}-\mathrm{P}_{\mathrm{sec}}\right) /\right.\right.$ $\left.\left.\left(\mathrm{P}_{\text {sat }}-\mathrm{P}_{\text {sec }}\right)\right]\right\}[12]$.

\subsubsection{Mesure de la transpiration}

Pour chaque porte-greffe de chaque traitement, une feuille, bien exposée et située sur la partie médiane de la plante, a été prélevée avec son pétiole à midi ; elle a été pesée 
immédiatement pour déterminer son poids frais initial $\left(\mathrm{P}_{1}\right)$. Après $60 \mathrm{~min}$ de transpiration à l'air libre, sous ombrage, et à $29,34^{\circ} \mathrm{C}$, son poids $\left(\mathrm{P}_{2}\right)$ a été de nouveau mesuré. La surface foliaire a été mesurée selon la formule [S $=(\pi \times a \times b) / 4]$ où $a$ et $b$ sont respectivement la longueur et la largeur du limbe $(\mathrm{cm})$. Pour les feuilles trifoliées, la surface foliaire a été évaluée par la somme de la surface des trois folioles. La transpiration $\left(\right.$ en $\left.\mathrm{g} \mathrm{H}_{2} \mathrm{O} \cdot \mathrm{cm}^{-2} \cdot \mathrm{s}^{-1}\right)$ a été calculée par la formule $\left[\mathrm{T}=\left(\mathrm{P}_{1}-\mathrm{P}_{2}\right) /\left(\mathrm{S}^{2} \times \mathrm{T}_{\text {trans }}\right)\right]$, où $\mathrm{T}_{\text {trans }}$ est la durée de la transpiration exprimée en secondes.

\subsubsection{Paramètres biochimiques}

Les taux de proline et de sucres solubles totaux ont été mesurés sur des échantillons prélevés sur les plants de chaque traitement et chaque porte-greffe. La quantité de chlorophylle totale a été mesurée également pour chaque traitement et chaque portegreffe avec un chlorophylle-mètre (type SPAD) sur les feuilles des plantes en pots.

\subsubsection{Dosage de la proline des feuilles}

La méthode de Monneveux et Nemmar a été utilisée pour le dosage de la proline [13]. Les valeurs obtenues ont été converties en teneur de proline $\left(\mu \mathrm{g} \cdot \mathrm{g}^{-1}\right.$ de matière sèche) à l'aide de l'équation $Y=0,1043 \times X$, où $X$ est la densité optique à $528 \mathrm{~nm}\left(\mathrm{DO}_{528 \mathrm{~nm}}\right)$.

\subsubsection{Dosage des sucres solubles totaux des feuilles}

Pour le dosage des sucres solubles totaux, nous avons utilisé la méthode de Dubois et al. [14]. Les valeurs obtenues ont été converties en teneur en mg de sucre soluble totaux par g de matière sèche à l'aide de l'équation de la courbe d'étalon : [ $Y=4,3918 \times(X-$ $0,194)]$, où $Y$ est la teneur en sucres solubles totaux et $X$ est la densité optique.

\subsubsection{Mesure de la chlorophylle totale des feuilles}

La chlorophylle totale des feuilles a été évaluée par un chlorophylle-mètre (type SPAD502, Konica-Minolta Sensing, INC., Japon). Les mesures ont été effectuées sur trois feuilles situées sur la partie médiane de la plante, pour chaque traitement et pour chaque porte-greffe [15].

\subsection{Analyses statistiques}

L'analyse de la variance (ANOVA) a été effectuée en utilisant la procédure GLM du logiciel statistique SAS (SAS Institute Inc., NC, U.S.A., version 9). Deux facteurs de classification ont été utilisés, le facteur dose d'irrigation et le facteur porte-greffe. La comparaison des moyennes a été faite par le test de la plus petite différence significative (LDS) au seuil de 0,05.

\section{Résultats}

\subsection{Effet du stress hydrique sur les paramètres morphologiques}

\subsubsection{Effet sur la hauteur de tige}

La hauteur de tige des porte-greffes d'agrumes testés a été fortement affectée par le régime hydrique imposé aux plants. L'analyse de la variance a montré un effet très hautement significatif du traitement $(P<0,0001)$ et du porte-greffe $(P=0,0001)$. Cependant, aucune interaction significative n'a été observée entre les porte-greffes et les traitements $(P=0,3214)$. Tous les portegreffes utilisés présentent donc un comportement similaire quant à la hauteur de leur tige vis-à-vis du régime d'irrigation appliqué.

La hauteur de la tige a diminué en même temps que le stress hydrique augmentait : à la dose de $100 \%$ Hcc, la hauteur de tige moyenne a été de $15 \mathrm{~cm}$, tous porte-greffes confondus; en revanche, à $50 \% \mathrm{Hcc}$, soit en condition de stress hydrique, la hauteur de tige a été de l'ordre de 9,37 cm. La comparaison des moyennes a permis de distinguer trois groupes homogènes constitués respectivement par les traitements d'irrigation à $(100 \%, \quad 75 \%$ et $50 \%)$ Hcc (tableau II).

Les porte-greffes présentant la tige la plus haute ont été Poncirus trifoliata $(15,62 \mathrm{~cm})$, le citrange Troyer $(14,92 \mathrm{~cm})$, le citrange 
Carrizo (France) $(14,52 \mathrm{~cm})$ et le citrange Carrizo (Maroc) $(14,13 \mathrm{~cm})$. Les portegreffes, Citrumelo et Citrus volkameriana ont été les moins vigoureux avec une hauteur finale de tige dépassant à peine $9,30 \mathrm{~cm}$ (tableau III).

\subsubsection{Effet sur les biomasses fraîche et sèche}

Les biomasses fraîche et sèche de différentes parties de la plante ont été nettement affectées par le stress hydrique. Les effets du régime hydrique et du porte-greffe sur les poids frais et sec des feuilles, tiges et racines ont été très hautement significatifs $(P<0,0003)$, alors qu'une interaction significative entre porte-greffe et régime hydrique n'a été révélée que pour le poids frais des feuilles $(P<0,0001)$. Le comportement des porte-greffes vis-à-vis des contraintes hydriques serait donc similaire en termes de poids frais et sec des feuilles, tige et racines des plants.

Pour les feuilles, les biomasse foliaires les plus élevées ont été produites par les portegreffes citrange Carrizo (Maroc), citrange Troyer, Citrus volkameriana et Poorman ; pour la tige, ce sont les porte-greffes

\section{Tableau II.}

Comparaison des moyennes de la hauteur de tige de jeunes portegreffes d'agrumes en fonction de différents traitements de déficit hydrique appliqués aux plants (dix porte-greffes confondus).

\begin{tabular}{lc} 
Régime hydrique $^{1}$ & $\begin{array}{c}\text { Hauteur de la tige } \\
(\mathrm{cm})\end{array}$ \\
\hline $\mathrm{T}_{0}(100 \% \mathrm{Hcc})$ & $15,00 \mathrm{a}$ \\
$\mathrm{T}_{1}(75 \% \mathrm{Hcc})$ & $12,55 \mathrm{~b}$ \\
$\mathrm{~T}_{2}(50 \% \mathrm{Hcc})$ & $9,36 \mathrm{c}$ \\
& \\
LSD & 1,6335
\end{tabular}

${ }^{1} \mathrm{Hcc}$ : humidité à la capacité au champ $\left(\mathrm{H}_{\mathrm{cc}}\right)$ du substrat.

Dans une même colonne, les moyennes suivies de lettres différentes sont significativement différentes $\left(\mathrm{LSD}_{0,05}\right)$.

citrange Carrizo (France), citrange Carrizo (Maroc) et citrange Troyer qui ont présenté les poids frais et sec les plus élevés ; pour les racines, les meilleurs poids frais et sec ont été obtenus également chez les portegreffes citrange Carrizo (France), citrange Carrizo (Maroc) et citrange Troyer (tableau III).

Tableau III.

Comparaison des moyennes de la hauteur de tige, ainsi que des poids frais et poids secs de la tige, des feuilles et des racines de jeunes porte-greffes d'agrumes soumis à différents traitements de déficit hydrique (tous traitements confondus).

\begin{tabular}{|c|c|c|c|c|c|c|c|}
\hline \multirow[t]{3}{*}{ Porte-greffe } & \multirow{3}{*}{$\begin{array}{l}\text { Hauteur de la tige } \\
\qquad(\mathrm{cm})\end{array}$} & \multicolumn{2}{|c|}{ Feuilles } & \multicolumn{2}{|c|}{ Tige } & \multicolumn{2}{|c|}{ Racines } \\
\hline & & Poids frais & Poids sec & Poids frais & Poids sec & Poids frais & Poids sec \\
\hline & & \multicolumn{6}{|c|}{ (g) } \\
\hline Citrange Carrizo (France) & $14,52 a b$ & $0,65 b$ & $0,19 \mathrm{bcd}$ & $0,52 \mathrm{a}$ & $0,24 a$ & $0,78 a b$ & $0,38 a b c$ \\
\hline Citrange Carrizo (Maroc) & $14,13 \mathrm{ab}$ & $0,72 a b$ & $0,22 a b$ & $0,58 a$ & $0,26 a$ & $0,91 \mathrm{a}$ & $0,43 a b$ \\
\hline Citrange Troyer & $14,92 a b$ & $0,73 a b$ & $0,22 a b$ & $0,57 \mathrm{a}$ & $0,27 \mathrm{a}$ & $0,79 a b$ & $0,45 \mathrm{a}$ \\
\hline Citrange Yuma & 10,82 ed & $0,46 \mathrm{c}$ & $0,13 \mathrm{ed}$ & $0,35 \mathrm{bc}$ & $0,18 \mathrm{bc}$ & $0,49 \mathrm{~cd}$ & 0,29 cde \\
\hline Citrumelo & $9,31 \mathrm{e}$ & $0,41 c$ & 0,12 e & $0,24 \mathrm{~d}$ & $0,13 d$ & $0,48 \mathrm{~cd}$ & 0,25 de \\
\hline Citrus volkameriana & 9,32 e & $0,70 a b$ & $0,20 \mathrm{bc}$ & $0,25 d$ & $0,11 d$ & $0,43 \mathrm{~cd}$ & 0,29 cde \\
\hline Mand., Sunki $\times$ Poncirus trifoliata & $12,09 \mathrm{~cd}$ & $0,48 \mathrm{c}$ & 0,14 cde & $0,25 d$ & $0,12 d$ & $0,32 \mathrm{~d}$ & $0,20 \mathrm{e}$ \\
\hline Poncirus trifoliata (I) & $15,62 \mathrm{a}$ & $0,41 c$ & 0,12 e & $0,40 \mathrm{~b}$ & $0,19 b$ & $0,60 \mathrm{bc}$ & $0,32 \mathrm{bcd}$ \\
\hline Poncirus trifoliata (II) & $13,33 \mathrm{bc}$ & $0,36 \mathrm{c}$ & 0,10 e & $0,32 \mathrm{bcd}$ & $0,14 \mathrm{~cd}$ & $0,60 \mathrm{bc}$ & $0,33 \mathrm{bcd}$ \\
\hline Poorman & 9,33 e & $0,83 a$ & 0,27 a & $0,30 \mathrm{~cd}$ & $0,15 \mathrm{~cd}$ & $0,59 \mathrm{bc}$ & $0,43 a b$ \\
\hline LSD & 1,6787 & 0,1444 & 0,0644 & 0,0919 & 0,0446 & 0,2069 & 0,1135 \\
\hline
\end{tabular}

Dans une même colonne, les moyennes suivies de lettres différentes sont significativement différentes $\left(\mathrm{LSD}_{0,05}\right)$. 


\begin{tabular}{|c|c|c|c|}
\hline Paramètres & Contenu relatif en eau & Taux de transpiration & Unités de SPAD ${ }^{1}$ \\
\hline Traitement (Trait) & $<0,0001$ & 0,0002 & $<0,0001$ \\
\hline Porte-greffes (PG) & 0,0281 & 0,002 & 0,0016 \\
\hline Interaction [Trait $\times$ PG] & 0,3150 & 0,9857 & 0,1648 \\
\hline
\end{tabular}

\section{Tableau V.}

Comparaison des moyennes des paramètres 'contenu relatif en eau', 'transpiration foliaire' et 'unités de SPAD' de jeunes porte-greffes d'agrumes soumis à divers traitements de déficit hydrique (tous traitements confondus).

\begin{tabular}{|c|c|c|c|}
\hline Porte-greffe & $\begin{array}{l}\text { Contenu relatif en eau } \\
\qquad(\%)\end{array}$ & $\begin{array}{l}\text { Transpiration foliaire } \\
\left(\mathrm{mg} \mathrm{H}_{2} \mathrm{O} \cdot \mathrm{cm}^{-2} \cdot \mathrm{s}^{-1}\right)\end{array}$ & Unités de SPAD ${ }^{1}$ \\
\hline Citrange Carrizo (France) & $72,32 \mathrm{bc}$ & $6,19 \mathrm{~b}$ & $35,07 \mathrm{a}$ \\
\hline Citrange Carrizo (Maroc) & $77,58 \mathrm{abc}$ & $15,49 \mathrm{a}$ & $27,93 \mathrm{bcd}$ \\
\hline Citrange Troyer & $75,86 \mathrm{bc}$ & $5,55 \mathrm{~b}$ & $32,53 a b$ \\
\hline Citrange yuma & $80,83 a b$ & $5,45 \mathrm{~b}$ & $30,5 \mathrm{abc}$ \\
\hline Citrumelo & $79,85 a b c$ & $5,31 \mathrm{~b}$ & $25,94 \mathrm{~cd}$ \\
\hline Citrus volkameriana & 88,49 a & $5,88 \mathrm{~b}$ & $32,26 \mathrm{ab}$ \\
\hline Mand. Sunki $\times$ Poncirus trifoliata & $83,37 \mathrm{ab}$ & $3,21 \mathrm{~b}$ & $28,72 \mathrm{bc}$ \\
\hline Poncirus trifoliata (I) & $68,32 \mathrm{c}$ & $6,21 \mathrm{~b}$ & $23,84 d$ \\
\hline Poncirus trifoliata (II) & $78,82 \mathrm{abc}$ & $4,84 \mathrm{~b}$ & $29,37 \mathrm{bc}$ \\
\hline Poorman & 88,02 a & $4,53 \mathrm{~b}$ & $29,69 \mathrm{bc}$ \\
\hline LSD & 11,925 & 5,13 & 4,9185 \\
\hline
\end{tabular}

\subsection{Effet du stress hydrique sur les paramètres physiologiques et biochimiques}

\subsubsection{Effet sur le contenu relatif en eau, le taux de la transpiration et la teneur des feuilles en chlorophylle}

Notre expérimentation a mis en évidence des effets très hautement significatifs du régime hydrique sur le contenu relatif en eau des porte-greffes, le taux de transpiration et la teneur des feuilles en chlorophylle. Cependant, aucune interaction entre ces différents paramètres et le facteur porte-greffes n'a été significative (tableau IV). Globalement, l'augmentation du stress hydrique appliqué aux plants a induit une diminution de ces paramètres (tableau $V$ ).

Pour la teneur relative en eau des feuilles, les porte-greffes Citrus volkameriana (88.49\%) et Poorman (88.02 \%) ont montré les valeurs les plus élevées alors que le porte-greffe Poncirus trifoliata (68.32 \%) a présenté la valeur la plus basse (tableau VI). Par ailleurs, le porte-greffe citrange Carrizo (Maroc) a affiché le plus fort taux de la transpiration $\left(15,49 \mathrm{mg} \mathrm{H} \mathrm{H}_{2} \mathrm{O} \cdot \mathrm{cm}^{-2} \cdot \mathrm{s}^{-1}\right)$, alors que les autres porte-greffes constituaient un deuxième groupe dont les taux ont varié de $(3,21$ à 6,25$) \mathrm{mg} \mathrm{H}_{2} \mathrm{O} \cdot \mathrm{cm}^{-2} \cdot \mathrm{s}^{-1}$ (tableau VI). Enfin, les feuilles du citrange Carrizo (France) ont présenté la teneur en chlorophylle la plus élevée (35,07 unités SPAD), 
Tableau VI.

Comparaison des moyennes entre différents régimes hydriques pour les paramètres 'contenu relatif en eau', 'transpiration foliaire' et 'unités de SPAD' de jeunes porte-greffes d'agrumes soumis à divers traitements de déficit hydrique (tous les porte-greffes confondus).

\begin{tabular}{lccc}
\hline Régime hydrique & $\begin{array}{c}\text { Contenu relatif en eau } \\
(\%)\end{array}$ & $\begin{array}{c}\text { Transpiration foliaire } \\
\left(\mathrm{mg} \mathrm{H}_{2} \mathrm{O} \cdot \mathrm{cm}^{-2} \cdot \mathrm{s}^{-1}\right)\end{array}$ & Unités de SPAD $^{2}$ \\
\hline $\mathrm{T}_{0}(100 \% \mathrm{Hcc})$ & $88,77 \mathrm{a}$ & $9,342 \mathrm{a}$ & $35,48 \mathrm{a}$ \\
$\mathrm{T}_{1}(75 \% \mathrm{Hcc})$ & $85,29 \mathrm{a}$ & $6,325 \mathrm{a}$ & $30,17 \mathrm{~b}$ \\
$\mathrm{~T}_{2}(50 \% \mathrm{Hcc})$ & $64,16 \mathrm{~b}$ & $3,142 \mathrm{~b}$ & $23,10 \mathrm{c}$ \\
LSD & 8,5567 & 5,1378 & 2,7115
\end{tabular}

${ }^{1}$ Hcc : humidité à la capacité au champ du substrat.

2 SPAD : instrument permettant de mesurer la teneur de la feuille en chlorophylle.

Dans une même colonne, les moyennes suivies de lettres différentes sont significativement différentes $\left(\operatorname{LSD}_{0,05}\right)$.

\section{Tableau VII.}

Teneur en sucres soluble $\left(\mathrm{mg} \cdot \mathrm{g}^{-1}\right.$ de matière sèche) des porte-greffes d'agrumes en fonction du niveau du stress hydrique appliqué aux plants.

\begin{tabular}{lccc}
\hline Porte-greffe & \multicolumn{2}{c}{ Régime hydrique $^{1}$} & $50 \% \mathrm{Hcc}$ \\
& $100 \% \mathrm{Hcc}$ & $75 \% \mathrm{Hcc}$ & $2,227 \mathrm{a}$ \\
Citrange Carrizo (France) & $0,380 \mathrm{~cd}$ & $0,725 \mathrm{a}$ & $1,769 \mathrm{~b}$ \\
Citrange Carrizo (Maroc) & $0,246 \mathrm{~d}$ & $0,760 \mathrm{a}$ & $1,556 \mathrm{c}$ \\
Citrange Troyer & $0,403 \mathrm{bcd}$ & $0,237 \mathrm{~d}$ & $0,842 \mathrm{f}$ \\
Citrange Yuma & $0,578 \mathrm{abc}$ & $0,545 \mathrm{bc}$ & $1,042 \mathrm{e}$ \\
Citrumelo & $0,610 \mathrm{ab}$ & $0,490 \mathrm{c}$ & $1,160 \mathrm{e}$ \\
Citrus volkameriana & $0,728 \mathrm{a}$ & $0,641 \mathrm{ab}$ & $1,604 \mathrm{c}$ \\
Mand. Sunki $\times$ Poncirus trifoliata & $0,250 \mathrm{bcd}$ & $0,443 \mathrm{c}$ & $1,590 \mathrm{c}$ \\
Poncirus trifoliata (I) & $0,451 \mathrm{bcd}$ & $0,250 \mathrm{~d}$ & $1,320 \mathrm{~d}$ \\
Poncirus trifoliata (II) & $0,459 \mathrm{bcd}$ & $0,499 \mathrm{c}$ & $0,762 \mathrm{f}$ \\
Poorman & $0,352 \mathrm{~d}$ & $0,473 \mathrm{c}$ & 0,126 \\
& & 0,1316 &
\end{tabular}

alors que les feuilles du porte-greffe Poncirus trifoliata montrait la teneur la plus basse (23,84 unités SPAD) (tableau VI).

\subsubsection{Effet sur l'accumulation des substances organiques}

L'application d'un stress hydrique a provoqué une augmentation de la teneur en substances organiques (sucres et proline) dans les feuilles des plants d'agrumes (tableaux VII, VIII). L'analyse de la variance relative à la teneur en sucres solubles totaux et en proline a montré un effet très hautement significatif du traitement $(P<0,0001)$, du porte-greffe $(P<0,0001)$ et de l'interaction [traitement $\times$ portegreffe] $(P<0,0001)$. 
Tableau VIII.

Teneur en proline en $\left(\mu \mathrm{g} \cdot \mathrm{g}^{-1}\right.$ de matière sèche) dans les feuilles de jeunes portegreffes d'agrumes en fonction du niveau du stress hydrique appliqué aux plants.

\begin{tabular}{lccc}
\hline Porte-greffe & \multicolumn{3}{c}{ Régime hydrique $^{1}$} \\
\cline { 2 - 4 } Citrange Carrizo (France) & $100 \% \mathrm{Hcc}$ & $75 \% \mathrm{Hcc}$ & $50 \% \mathrm{Hcc}$ \\
Citrange Carrizo (Maroc) & $11,058 \mathrm{f}$ & $12,343 \mathrm{f}$ & $48,578 \mathrm{a}$ \\
Citrange Troyer & $14,911 \mathrm{e}$ & $17,300 \mathrm{ef}$ & $35,237 \mathrm{e}$ \\
Citrange Yuma & $10,234 \mathrm{f}$ & $21,230 \mathrm{de}$ & $49,129 \mathrm{a}$ \\
Citrumelo & $24,874 \mathrm{~cd}$ & $28,184 \mathrm{c}$ & $35,931 \mathrm{e}$ \\
Citrus volkameriana & $31,138 \mathrm{ab}$ & $18,811 \mathrm{e}$ & $41,013 \mathrm{~cd}$ \\
Mand. Sunki $\times$ Poncirus trifoliata & $32,923 \mathrm{a}$ & $33,875 \mathrm{~b}$ & $49,053 \mathrm{a}$ \\
Poncirus trifoliata (I) & $20,624 \mathrm{~d}$ & $18,249 \mathrm{e}$ & $44,391 \mathrm{bc}$ \\
Poncirus trifoliata (II) & $27,330 \mathrm{bc}$ & $25,040 \mathrm{~cd}$ & $37,375 \mathrm{de}$ \\
Poorman & $15,619 \mathrm{e}$ & $25,646 \mathrm{~cd}$ & $41,357 \mathrm{c}$ \\
& $31,843 \mathrm{ab}$ & $45,168 \mathrm{a}$ & $47,876 \mathrm{ab}$ \\
LSD & 4,6107 & & 3,9453 \\
\hline & & 5,2639 & \\
Dans : humidité à la capacité au champ du substrat. & & \\
différentes (LSD & &
\end{tabular}

Pour les plants sous régime hydrique à $50 \%$ Hcc, la teneur des feuilles en sucres solubles de la plupart des porte-greffes testés a été plus que doublée par rapport aux plants irrigués à $100 \%$ Hcc (tableau VII). Sous régime hydrique favorable (100\% Hcc), la teneur en sucre la plus élevée a été notée chez le porte-greffe Citrus volkameriana $\left(0,728 \mathrm{mg}\right.$ sucre $\left.\cdot \mathrm{g}^{-1} \mathrm{~ms}\right)$. En revanche, sous régime de stress à $50 \%$ Hcc, c'est le porte-greffe citrange Carrizo (France) qui a enregistré la valeur maximale $(2,227 \mathrm{mg}$ de sucre $\mathrm{g}^{-1} \mathrm{~ms}$ ) (tableau VII).

Sous le régime hydrique le plus stressant (50\% Hcc), la teneur la plus élevée en proline a été enregistrée par les porte-greffes citrange Troyer $\left(49,129 \mu \mathrm{g}\right.$ proline $\left.\cdot \mathrm{g}^{-1} \mathrm{~ms}\right)$, Citrus volkameriana (49,053 $\mathrm{\mu g}$ proline $\cdot \mathrm{g}^{-1}$ $\mathrm{ms})$, citrange Carrizo (France) $(48,578 \mu \mathrm{g}$ proline $\left.\cdot \mathrm{g}^{-1} \mathrm{~ms}\right)$ et Poorman $(47,876 \mu \mathrm{g}$ proline $\cdot \mathrm{g}^{-1} \mathrm{~ms}$ ). La teneur en proline la plus faible a été enregistrée pour les portegreffes citrange Carrizo (Maroc) $(35,237 \mu \mathrm{g}$ proline $\left.\cdot \mathrm{g}^{-1} \mathrm{~ms}\right)$ et citrange Yuma $(35,931 \mu \mathrm{g}$ proline $\cdot \mathrm{g}^{-1} \mathrm{~ms}$ ). Sous régime hydrique à $100 \%$ Hcc, les porte-greffes Citrus volkame- riana citrumelo et Poorman ont accumulé la teneur la plus élevée en proline (autour de 31 ug.g ms) (tableau VIII).

\section{Discussion}

Les plantes sont susceptibles d'être exposées à divers stress biotiques ou abiotiques qui influent sur leur développement, leur croissance et leur productivité. Un des principaux stress abiotiques affectant la productivité des plantes est le stress hydrique dû à la sécheresse et, sous certaines conditions, à une augmentation de la concentration de sel dans le sol [16]. Le degré de résistance des plants vis-à-vis de tels stress est variable. De ce fait, une bonne compréhension des changements morphologiques, physiologiques et biochimiques de ces plants sous conditions de stress hydrique pourrait être utile dans des programmes de création ou de sélection de nouvelles variétés visant à améliorer leur productivité lorsque soumises à de telles conditions $[17,18]$. 
Dans notre étude, l'application d'un stress hydrique à de jeunes plants de portegreffes d'agrumes a provoqué une réduction significative de la hauteur finale des plants, variable en fonction du porte-greffe considéré. Pour ce paramètre de croissance, les citranges que nous avons testés ont montré les meilleures aptitudes de tolérance au stress hydrique par rapport aux autres portegreffes utilisés. Le citrumelo et l'hybride Mand. Sunki $\times$ Poncirus trifoliata se sont montrés les plus sensibles. Des résultats similaires ont été enregistrés pour d'autres cultures. En effet, chez le soja, la croissance en longueur des tiges diminuerait également en conditions de stress hydrique [19]. Par ailleurs, Wu et al. ont observé aussi une réduction importante de la croissance en longueur de jeunes plants d'agrumes [20] et, chez la pomme de terre, la croissance en longueur des tiges serait de même significativement affectée par le stress hydrique [21].

Le poids de matières sèche et fraîche des différents porte-greffes a diminué simultanément avec l'intensité du stress hydrique appliqué. Cette réduction a été très sévère chez les porte-greffes très sensibles tel que citrumelo et Poncirus trifoliata, ce qui serait en accord avec les travaux de Saxena and Nautiyal [22], Fu and Huang [23] et Monti et al. [24]. Une telle réduction de la production de la matière sèche sous conditions de stress hydrique a également été rapportée chez différentes autres cultures telles que le peuplier [25], le soja [26] et d'autres espèces [27].

Nos expérimentations ont également mis en évidence que le taux de transpiration des feuilles de la plupart des porte-greffes placés en conditions de stress hydrique diminuait en même temps qu'augmentait la sévérité du stress appliqué. En effet, le taux de transpiration le plus élevé a été observé chez le porte-greffe citrange Carrizo (Maroc) sous le régime à $50 \%$ Hcc. Selon Tezara et al., la diminution de la transpiration serait due à une diminution de la conductance des stomates et, par conséquent, à une diminution de la photosynthèse [28]. Dans ce sens, le citrange Carrizo (Maroc) pourrait être considéré comme tolérant au déficit hydrique puisqu'il est capable de maintenir un niveau de transpiration élevé sous un régime hydrique de $50 \%$ Hcc. Cela pourrait être en relation avec la capacité de ce portegreffe à maintenir ses activités de photosynthèse qui, en améliorant l'absorption du $\mathrm{CO}_{2}$, favorise l'accumulation de sucres et d'osmolytes permettant de maintenir la turgescence des cellules de garde des stomates sous des conditions de stress hydriques.

Chez les plantes, des changements importants des ratios en chlorophylles $a$ et $b$ ainsi que des caroténoïdes sont observés lorsqu'elles sont placées en conditions de stress hydrique [27, 29-31]. Dans notre étude, nous avons noté que la teneur en chlorophylle exprimée en unités de SPAD diminuait simultanément avec l'intensité du stress hydrique imposé. De la même façon, une réduction importante de la teneur en chlorophylle a été notée chez le cotonnier sous conditions de stress hydrique [32], ainsi que chez Catharanthus roseus [8], Helianthus annuus (tournesol) [31] et Vaccinium myrtillus [33]. Les valeurs maximales de la teneur en chlorophylles enregistrées chez certains porte-greffes d'agrumes que nous avons testés pourraient être en relation avec le degré de résistance de ces pigments à la déshydratation chez ces mêmes portegreffes. Dans ce sens, Luvaha et al. ont rapporté que, sous stress hydrique, il pouvait y avoir une faible augmentation de la teneur en chlorophylle $a$ comparée à celle de la teneur en chlorophylle $b$ qui resterait constante [4].

La teneur en proline a augmenté différemment selon les porte-greffes que nous avons étudiés. Dans ce contexte, le citrange Carrizo (France), le citrange Troyer et Poorman ont affiché les valeurs maximales sous un régime hydrique à $50 \% \mathrm{Hcc}$; les portegreffes à faibles teneurs en proline sous les mêmes conditions (50\% Hcc) ont été le citrange Carrizo (Maroc) et le citrange Troyer.

Pour de nombreuses plantes, l'accumulation de la proline a été fréquemment évoquée comme réponse à une variété de stress abiotiques [34, 35]. Toutefois, le rôle précis de l'accumulation de cet acide aminé n'est pas encore élucidé. Il pourrait agir comme osmo-régulateur [36], osmo-protecteur [37], ou régulateur de l'oxydoréduction potentiel des cellules [38]. L'accumulation élevée de proline enregistrée chez certains portegreffes testés dans notre étude, notamment 
chez Poorman, pourrait être en relation avec leur degré de tolérance au déficit hydrique [39].

Dans notre étude, la teneur en sucres des jeunes plants a été également augmentée sous l'effet d'un stress hydrique. Le contenu en sucres solubles le plus élevé a été observé chez le porte-greffe citrange Carrizo (France), alors que le plus faible était enregistré pour Poorman.

L'augmentation de la concentration en sucres pourrait être le résultat de la dégradation de l'amidon dans les feuilles de porte-greffes placés sous conditions de stress hydrique, ce qui permettrait à la plante de maintenir de l'eau au niveau de la cellule suite à une augmentation du potentiel osmotique intracellulaire [40]. Dans ce sens, il a été rapporté que l'accumulation de sucres solubles était fortement corrélée à l'acquisition de tolérance à la sécheresse chez les plantes [41].

Finalement, sur la base des résultats que nous avons obtenus, ce sont les portegreffes citrange Carrizo (Maroc) et Poorman qui présenteraient globalement les meilleures aptitudes à résister aux conditions de stress hydrique.

\section{Références}

[1] Vanderweyen A., Contribution à l'étude de la gommose à Phytophthora des agrumes au Maroc, Univ. Nancy, Thèse, Nancy, France, 1982, $160 \mathrm{p}$.

[2] Benyahia H., Ait Haddou M.M., Jrifi A., Lamsettef $Y$., Effet de la salinité de l'eau d'irrigation sur la colonisation des racines des porte-greffes d'agrumes par Phytophthora parasitica, Fruits 59 (2004) 101-108.

[3] Maqsood M., Azam Ali S.N., Effects of drought on growth, development, radiation use efficiency and yield of finger millet (Eleucine coracana), Pak. J. Bot. 39 (1) (2007) 123-134.

[4] Luvaha E., Netondo G.W., Ouma G., Effect of water deficit on the physiological and morphological characteristics of mango (Mangifera indica) rootstocks seedlings, Am. J. Plant Physiol. 3 (1) (2008) 1-15.
[5] Doorenbos J., Kassam A.H., Yield responses to water, FAO irrigation and drainage paper no 33, FAO, Roma, Italy, 1979.

[6] Yonemoto Y., Matsumoto K., Furukawa T., Asakawa M., Okuda H., Takahara T., Effects of rootstock and crop load on sap flow rate in branches of 'Shirakawa Satsuma' mandarin (Citrus unshiu Marc.), Sci. Hortic. 102 (2004) 295-300.

[7] Chaves M.M., Pereira J.S., Maroco J., Rodriques M.L., Ricardo C.P.P., Osorio M.L., Carvatho I., Faria T., Pinheiro C., How plants cope with water stress in the field photosynthesis and growth?, Ann. Bot. 89 (2002) 907-916.

[8] Jaleel C.A., Manivannan P., Lakshmanan G.M.A., Gomathinayagam M., Panneerselvam R., Alterations in morphological parameters and photosynthetic pigment responses of Catharanthus roseus under soil water deficits, Colloids Surf. B: Biointerfaces 61 (2008) 298-303.

[9] Jaleel C.A., Manivannan P., Wahid A., Farooq M., Somasundaram R., Panneerselvam R., Drought stress in plants: a review on morphological haracteristics and pigments composition, Int. J. Agric. Biol. 11 (2009) 100105.

[10] Reddy A.R., Chaitanya K.V., Vivekanandan M., Drought induced responses of photosynthesis and antioxidant metabolism in higher plants, J. Plant Physiol. 161 (2004) 1189-1202.

[11] Zhao C.X., Guo L.Y., Jaleel C.A., Shao H.B., Yang H.B., Prospects for dissecting plant adaptive molecular mechanisms to improve wheat cultivars in drought environments, Comp. Rend. Biol. 331 (2008) 579-586.

[12] Siddiqi E.H., Ashraf M., Can leaf water relation parameters be used as selection criteria for salt tolerance in safflower (Carthamus tinctorius L.), Pak. J. Bot. 40 (1) (2008) 221228.

[13] Monneveux P.H., Nemmar M., Contribution à l'étude de la résistance à la sécheresse chez le blé tendre (Triticum aestivum L.) et chez le blé dur (Triticum durum Desf.) : Étude de l'accumulation de la proline au cours du cycle de développement, Agronomie 6 (6) (1986) 583-590.

[14] Dubois M., Gilles K.A., Hamilton J.K., Rebers P.A., Smith E., Colorimetric method for determination of sugar and related substances, Allal. Chem. 28 (1956) 350-356. 
[15] Guénette C., Évaluation du potentiel de l'infrarouge spectral pour la détection hâtive du stress nutritif chez les végétaux de grandes cultures : application à la culture de la pomme de terre, Fac. For. Géomat., Univ. Laval Québec, Thèse, Laval, Canada, 2003, $106 \mathrm{p}$.

[16] Gueta-Dahan Y., Yaniv Z., Zilinskas A., Ben Hayyim G., Salt and oxidative stress; similar and specific responses and their relation to salt tolerance in Citrus, Planta 203 (1997) 460-469.

[17] Nam N.H., Chauhan Y.S., Johansen C., Effect of timing of drought stress on growth and grain yield of extra-short-duration pigeonpea lines, J. Agric. Sci. 136 (2001) 179-189.

[18] Martinez J.P., Silva H., Ledent J.F., Pinto M., Effect of drought stress on the osmotic adjustment, cell wall elasticity and cell volume of six cultivars of common beans (Phaseolus vulgaris L.), Eur. J. Agron. 26 (2007) 30-38.

[19] Specht J.E., Chase K., Macrander M., Graef G.L., Chung J., Markwell J.P., Germann M., Orf J.H., Lark K.G., Soybean response to water. A QTL analysis of drought tolerance, Crop Sci. 41 (2001) 493-509.

[20] Wu Q.S., Xia R.X, Zou Y.N., Improved soil structure and citrus growth after inoculation with three arbuscular mycorrhizal fungi under drought stress, Eur. J. Soil Biol. 44 (2008) 122-128.

[21] Heuer B., Nadler A., Growth, development and yield of potatoes under salinity and water deficit, Aust. J. Agric. Res. 46 (1995) 1477-1486.

[22] Saxena, R., Nautiyal S., Variation in growth and survival of five seed-sources of Pinus roxburchii Sarg. under various stages of water stress, Plant Physiol. 5 (2001) 563573.

[23] Fu J., Huang B., Involvement of antioxidants and lipid peroxidation in the adaptation of two-cool season grasses to localized drought stress, Environ. Exp. Bot. 45 (2001) 105-114.

[24] Monti A., Amaducci M.T., Pritoni G., Verturi G., Variation in carbon isotope discrimination during growth and at different organs in sugar beet (Beta vulgaris L.), Field Crop Res. 98 (2006) 157-163.
[25] Wullschleger S.D., Yin T.M., DiFazio S.P., Tschaplinski T.J., Gunter L.E., Davis M.F., Tuskan G.A., Phenotypic variation in growth and biomass distribution for two advancedgeneration pedigrees of hybrid poplar, Can. J. For. Res. 35 (2005) 1779-1789.

[26] Zhang M., Duan L., Zhai Z., Li J., Tian X., Wang B., He Z., Li Z., Effects of plant growth regulators on water deficit-induced yield loss in soybean, Proc. 4th Int. Crop Sci. Congr., Brisbane, Australia, 2004.

[27] Farooq M., Wahid A., Kobayashi N., Fujita D., Basra S.M.A., Plant drought stress: effects, mechanisms and management, Agron. Sustain. Dev. 29 (2009) 185-212.

[28] Tezara W., Mitchel V., Driscul S.P., Lawlor D.W., Effects of water deficit and its interaction with $\mathrm{CO}_{2}$ supply on the biochemistry and physiology of photosynthesis in sunflower, Exp. Bot. 53 (2002) 1781-1791.

[29] Anjum F., Yaseen M., Rasul E., Wahid A., Anjum S., Water stress in barley (Hordeum vulgare L.). I. Effect on morphological characters, Pak. J. Agric. Sci. 40 (2003) 43-44.

[30] Havaux M., Carotenoids as membrane stabilizers in chloroplasts, Trends Plant Sci. 3 (1998) 147-151.

[31] Kiani S.P., Maury P., Sarrafi A., Grieu P., QTL analysis of chlorophyll fluorescence parameters in sunflower (Helianthus annuus L.) under well-watered and water-stressed conditions, Plant Sci. 175 (2008) 565-573.

[32] Massacci A., Nabiev S.M., Pietrosanti L., Nematov S.K., Chernikova T.N., Thor K., Leipner J., Response of the photosynthetic apparatus of cotton (Gossypium hirsutum) to the onset of drought stress under field conditions studied by gas exchange analysis and chlorophyll fluorescence imaging, Plant Physiol. Biochem. 46 (2008) 189-195.

[33] Tahkokorpi M., Taulavuori K., Laine K., Taulavuori E., After effects of drought-related winter stress in previous and current year stems of Vaccinium myrtillus L., Environ. Exp. Bot. 61 (2007) 85-93.

[34] Hare P.D., Cress W.A., Metabolic implications of stress-induced proline accumulation in plants, Plant Growth Regul. 21 (1997) 79102.

[35] Voetberg G.S., Sharp R.E., Growth of maize primary root at low water potential. III. Roles of increased proline deposition in osmotic 
adjustment, Plant Physiol. 96 (1991) 11251230.

[36] Delauney A.J., Verma D.P.S., Proline biosynthesis and osmoregulation in plants, Plant J. 4 (1993) 215-223.

[37] Csonka L.N., Physiological and genetic responses of bacteria to osmotic stress, Microbiol. Rev. 53 (1989) 121-147.

[38] Bellinger Y., Larher F., Proline accumulation in higher plants: A redox buffer?, Plant Physiol. 6 (1987) 23-27.
[39] Nayer M., Reza H., Drought-induced accumulation of soluble sugars and proline in two maize varieties, World Appl. Sci. J. 3 (3) (2008) 448-453.

[40] Fischer C., Höll W., Food reserves in Scots pine (Pinus sylvestris L.). I. Seasonal changes in the carbohydrate and fat reserves of pine needles, Trees 5 (1991) 187195.

[41] Hoekstra F.A., Buitink J., Mechanisms of plant dessiccation tolerance, Trends Plant Sci. 8 (6) (2001) 431-438.

\section{Evaluación de la resistencia de diez patrones de cítricos, resistentes a la tristeza, en relación con el déficit hídrico.}

Resumen - Introducción. En Marruecos, los cítricos están enfrentados a una combinación de estreses bióticos [virus de la tristeza de los cítricos (VTC) / Phytophthora] y abióticos (salinidad, alcalinidad y sequía). La mayoría de estas limitaciones debe gestionarse individualmente, mediante la elección del patrón adaptado. Sin embargo, la propagación del VTC en la cuenca mediterránea pronto impedirá el uso del naranjo agrio, el patrón tradicional, el cual, actualmente, ofrece una tolerancia suficiente a la salinidad y a la alcalinidad. Consecuentemente, la selección de nuevos patrones de cítricos se vuelve cada vez más urgente. Nuestro estudio pretendió estudiar el efecto de diferentes regímenes hídricos en el crecimiento de diez patrones de cítricos: citranjo Carrizo (Francia), citranjo Carrizo (Marruecos), citranjo Troyer, citranjo Yuma, Citrus volkameriana; dos Poncirus trifoliata de orígenes diferentes, citrumelo, Mand. Sunki $\times$ Poncirus trifoliata, y Poorman. Material y métodos. Para cada patrón, se cultivaron plantas de tres meses de edad en tiestos de plástico $(0,5 \mathrm{~L})$, en un invernadero en El Menzeh, INRA, Marruecos; fueron sometidas a tres regímenes hídricos [(100 \%, $75 \%$ y $50 \%$ ) de humedad a la capacidad de campo (Hcc) del sustrato]. La experimentación se dispuso en bloques subdivididos en tres tratamientos. Los parámetros morfológicos medidos fueron la altura de la planta y la acumulación de la materia seca y fresca. Los parámetros fisiológicos estudiados fueron el contenido relativo de agua, el índice de transpiración y los contenidos de clorofila, azúcares solubles y prolina. Resultados. Los parámetros morfológicos y fisiológicos se vieron afectados por la intensidad del estrés hídrico aplicado. Sin embargo, los patrones se comportaron diferentemente, en relación con esta limitación. El estrés hídrico indujo un aumento del contenido de prolina y de azúcares solubles, a nivel de las hojas de los patrones. Conclusión. El estrés hídrico afecta, de manera significativa, las características fisiológicas y morfológicas de los patrones de cítricos; y, en condiciones de déficit hídrico extremo $(50 \% \mathrm{Hcc})$, se constató un comportamiento diferencial de los patrones. En base a los resultados que obtuvimos, los patrones de citranjo Carrizo (Marruecos) y de Poorman serían los que presentarían, en general, las mejores aptitudes para resistir a las condiciones de estrés hídrico. Marruecos / Citrus / portainjertos / estrés de sequia / resistencia a la sequía /
prolina / contenido de carbohidratos 